\title{
Gene Discovery through Germline Whole Exome Sequencing in Patients with Endometriosis- Associated Ovarian Cancer \\ $\mathbb{A}_{\text {Austin Health }}$
}

PeterMac

Eryn Dow ${ }^{1,2}$ Yoland Antill1,3 Deepak Subramanian ${ }^{4}$ ViP Investigators ${ }^{1,4}$ Ian Campbell Paul James $^{1}$

${ }^{1}$ Parkville Familial Cancer Centre, Peter MacCallum Cancer Centre \& Royal Melbourne Hospital, Melbourne, Victoria ${ }^{2}$ Clinical Genetics Department, Austin Health, Melbourne,

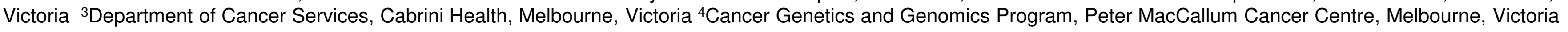

\section{BACKGROUND}

Endometriosis is common, affecting $5-25 \%$ of women of reproductive age ${ }^{1}$. Its aetiology remains poorly understood, but is likely multifactorial, including hormonal, immunological, and genetic factors ${ }^{1}$. Endometriosis has an estimated heritability of $51 \%$, and shares several characteristics with malignancy, including a capacity for invasion ${ }^{1}$; it is associated with an increased risk of endometrioid and clear cell ovarian cancer $^{1}$. Up to $60 \%$ of the underlying familial risk of ovarian cancer remains unexplained by high risk genes. In particular, the rate of germline BRCA1/2 mutations in endometrioid and clear cell ovarian cancers is low ${ }^{2}$, likely reflecting the different pathogenesis of these cancers and suggesting differing genetic predispositions in this group ${ }^{3}$. Our study aims to identify the novel germline genetic predispositions to endometrioid and clear cell endometriosis-associated ovarian cancer (EAOC).

\section{METHODS}

Women from the Variants in Practice (ViP) Study, which enrols women with ovarian cancer and uninformative $B R C A 1 / 2$ germline results for further genetic testing, were selected on the basis of having clear cell or endometrioid ovarian cancer arising within an endometriotic focus. Germline whole exome sequencing (WES) was performed using nextgeneration platforms (Agilent SureSelect capture, Illumina HiSeq 2500). Potential loss of function (LoF) variants (frameshift, stop-gain, in/del, essential splice site) were annotated through an established bioinformatics pipeline; annotated data were filtered to exclude artefactual, common (allele frequency $<0.005$ ) and likely irrelevant variants (pseudogenes and highly polymorphic genes including immunoglobulins and smell/taste receptors). LoF variant frequencies per gene were calculated and compared to publically available control population data $\left(G n o m A D^{4}\right)$ to identify genes of interest with an increased ratio of LoF germline variants per gene in the EAOC cohort. Further characterisation based on Emsembl Biotype (protein-coding) and published expression and functional data (Table 1) from PubMatrix ${ }^{5}$ multiplex literature mining identified promising gene candidates (Table 2).

TABLE 1 Summary of functional and expression data in the literature for candidate genes

\begin{tabular}{|c|c|c|c|c|c|c|c|}
\hline \multirow[t]{2}{*}{ Symbol } & \multirow{2}{*}{$\begin{array}{l}\text { Theoretical } \\
\text { Tumour } \\
\text { Suppressor Gene }\end{array}$} & \multicolumn{2}{|c|}{$\begin{array}{l}\text { Evidence of Tumour } \\
\text { Suppressor Activity } \\
\text { in any Malignancy }\end{array}$} & \multirow{2}{*}{$\begin{array}{l}\text { Evidence of } \\
\text { Expression in } \\
\text { Ovarian Tissues }\end{array}$} & \multicolumn{2}{|c|}{$\begin{array}{l}\text { Evidence of Tumour } \\
\text { Suppressor Activity } \\
\text { in Ovarian Cancer }\end{array}$} & \multirow{2}{*}{$\begin{array}{l}\text { Evidence of a } \\
\text { Functional Role in } \\
\text { Endometriosis }\end{array}$} \\
\hline & & in vitro & in vivo & & in vitro & in vivo & \\
\hline BRE & $\checkmark$ & $\checkmark$ & $\checkmark$ & $\checkmark$ & & & r \\
\hline FOXD3 & $\checkmark$ & $\checkmark$ & $\checkmark$ & $\checkmark$ & & & $\checkmark$ \\
\hline SALL2 & $\checkmark$ & $\checkmark$ & $\checkmark$ & $\checkmark$ & $\checkmark$ & $\checkmark$ & \\
\hline NRIP1 & $\checkmark$ & & $\checkmark$ & $\checkmark$ & & & $\checkmark$ \\
\hline ТОРЗВ & $\checkmark$ & & & $\checkmark$ & & & \\
\hline MRE11A & $\checkmark$ & & & $\checkmark$ & & $\checkmark$ & \\
\hline PARVB & $\checkmark$ & $\checkmark$ & $\checkmark$ & & & $\checkmark$ & \\
\hline CGN & $\checkmark$ & $\checkmark$ & & $\checkmark$ & & & \\
\hline XAF1 & $\checkmark$ & $\checkmark$ & $\checkmark$ & $\checkmark$ & $\checkmark$ & $\checkmark$ & \\
\hline MYO18B & $\checkmark$ & $\checkmark$ & $\checkmark$ & $\checkmark$ & $\checkmark$ & $\checkmark$ & \\
\hline TP53/3 & $\checkmark$ & $\checkmark$ & $\checkmark$ & & & $\checkmark$ & \\
\hline ENDOG & $\checkmark$ & & & & & & \\
\hline TNFRSF1OC & $\checkmark$ & $\checkmark$ & $\checkmark$ & $\checkmark$ & & $\checkmark$ & \\
\hline DLEC1 & $\checkmark$ & & $\checkmark$ & $\checkmark$ & $\checkmark$ & $\checkmark$ & \\
\hline
\end{tabular}

\section{REFERENCES}

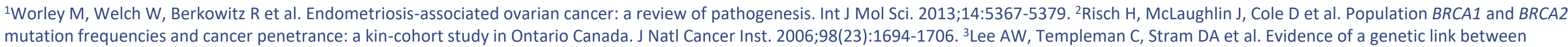
mutation frequencies and cancer penetrance: a kin-cohort study in Ontario Canada. J Natl Cancer Inst. 2006;98(23):1694-1706. ${ }^{3}$ Lee AW, Templeman C, Stram DA et al. Evidence of a genetic link between
endometriosis and ovarian cancer. Fertil Steril. 2016;105(1):35-43 ${ }^{4}$ Lek M, Karczewski KJ, Minikel EV et al. Analysis of protein-coding genetic variation in 60,706 humans. Nature. 2016;536:285. ${ }^{5}$ Becker KG, Dennis Jr G et al. PubMatrix: a tool for multiplex literature mining. BMC Bioinformatics. 2003;4:61.

\section{FIGURE 1}

610 patients in ViP with ovarian cancer and no $B R C A 1 / 2$ gene mutations

64 patients with histopathological evidence of endometriosis

25 patients with endometriosis adjacent to or directly contacting clear cell or endometrioid cancer

23 patients with adequate DNA available to proceed with whole exome sequencing

TABLE 2 Characteristics of candidate variants prioritised for further investigation

\begin{tabular}{|c|c|c|c|c|c|c|c|}
\hline Symbol & $\begin{array}{l}\text { HGSV Coding } \\
\text { Sequence Variant }\end{array}$ & dbSNP ID & $\begin{array}{l}\text { Variant } \\
\text { Classification }\end{array}$ & Pathology & Ob & $\begin{array}{l}\text { GnomAD } \\
\text { Frequency }\end{array}$ & $\begin{array}{l}\text { LoF } \\
\text { Ratio }\end{array}$ \\
\hline$B R E$ & $\begin{array}{l}\text { ENST00000361704.2: } \\
\text { c. } 1089-2 A>C\end{array}$ & rs150302537 & splice acceptor & Endometrioid & 2 & 0.00297 & 306.10 \\
\hline FOXD3 & $\begin{array}{l}\text { ENST00000371116.2: } \\
\text { c.127delG }\end{array}$ & $\mathrm{N} / \mathrm{A}$ & frameshift & $\begin{array}{l}\text { Mixed Clear Cell/ } \\
\text { Endometrioid }\end{array}$ & 1 & Not Reported & 237.90 \\
\hline SALL2 & $\begin{array}{l}\text { ENST00000327430.3: } \\
\text { c. } 2068 C>T\end{array}$ & N/A & stop gained & Endometrioid & 1 & Not Reported & 105.52 \\
\hline NRIP1 & $\begin{array}{l}\text { ENST00000318948.4: } \\
\text { c. } 916 G>T\end{array}$ & N/A & stop gained & Endometrioid & 1 & Not Reported & 52.42 \\
\hline TOP3В & $\begin{array}{l}\text { ENST00000357179.5: } \\
\text { c. } 470 \text { delA }\end{array}$ & rs768785125 & frameshift & Endometrioid & 1 & $4.07 \times 10^{-6}$ & 37.36 \\
\hline MRE11A & $\begin{array}{l}\text { ENST00000323977.3: } \\
\text { c.1726C>T }\end{array}$ & rs774277300 & stop gained & Endometrioid & 1 & $2.84 \times 10^{-5}$ & 33.38 \\
\hline PARVB & $\begin{array}{l}\text { ENST00000404989.1: } \\
\text { c.92-1_93delGAG }\end{array}$ & rs748653684 & splice acceptor & Endometrioid & 1 & 0.000152 & 32.93 \\
\hline CGN & $\begin{array}{l}\text { ENST00000271636.7: } \\
\text { c.333_334insC }\end{array}$ & $\mathrm{N} / \mathrm{A}$ & frameshift & Endometrioid & 1 & Not Reported & 14.15 \\
\hline XAF1 & $\begin{array}{l}\text { ENST00000361842.3: } \\
\text { c. } 32+1 G>A\end{array}$ & rs768435132 & splice donor & Endometrioid & 1 & $8.15 \times 10^{-6}$ & 11.17 \\
\hline MYO18B & $\begin{array}{l}\text { ENST00000335473.7: } \\
\text { c.3209-1delG }\end{array}$ & N/A & splice acceptor & Endometrioid & 1 & Not Reported & 8.46 \\
\hline TP5313 & $\begin{array}{l}\text { ENST00000238721.4: } \\
\text { c. } 772 C>T\end{array}$ & rs139719848 & stop gained & Endometrioid & 1 & 0.000108 & 8.37 \\
\hline ENDOG & $\begin{array}{l}\text { ENST00000372642.4: } \\
\text { c.886_889delAGTA }\end{array}$ & rs199801162 & frameshift & Endometrioid & 1 & 0.00220 & 7.87 \\
\hline TNFRSF1OC & $\begin{array}{l}\text { ENST00000356864.3: } \\
\text { c.269delT }\end{array}$ & rs537005361 & frameshift & Endometrioid & 1 & 0.00290 & 6.91 \\
\hline DLEC1 & $\begin{array}{l}\text { ENST00000452631.2: } \\
\text { c.5074_5075insCAAA }\end{array}$ & rs577236284 & frameshift & Endometrioid & 1 & 0.00252 & 4.80 \\
\hline
\end{tabular}

dbSNP ID; database of single nucleotide polymorphism identification, HGSV; Human Genome Variation Society, Ob; number of times variant is observed in the EAOC cohort, LoF; loss of function
obs

\section{CONCLUSION \& FUTURE DIRECTIONS}

The underlying genetic factors influencing EAOC risk differ from those in serous ovarian cancer. Using this highly curated population, preliminary results identified 14 candidate genes with an increased ratio of LoF variants per gene compared to control populations and a potential functional relevance to endometriosis and/or ovarian cancers. These candidate genes provide insight into the genetic contributions to EAOC but require further study before any definitive associations can be concluded. We plan to expand the cohort in a future study to include more cases of EAOC, as well as a control cohort of women with endometriosis but no EAOC. Additionally, nontruncating but potentially deleterious missense variants from the existing WES data are yet to be examined and may add further candidate genes. Validation of candidate genes, including confirmation of loss of the wildtype allele in tumour tissue, is still required. 